Purdue University

Purdue e-Pubs

CTRC Research Publications

Cooling Technologies Research Center

4-26-2004

\title{
Low Reynolds Number Flow Through Nozzle- Diffuser Elements in Valveless Micropumps
}

Vishal Singhal

Jayathi Y. Murthy

S V. Garimella

Purdue University, sureshg@purdue.edu

Follow this and additional works at: http:/ / docs.lib.purdue.edu/coolingpubs

Part of the Mechanical Engineering Commons

Singhal, Vishal; Murthy, Jayathi Y.; and Garimella, S V., "Low Reynolds Number Flow Through Nozzle-Diffuser Elements in Valveless Micropumps" (2004). CTRC Research Publications. Paper 17.

http://dx.doi.org/10.1016/j.sna.2004.03.002

This document has been made available through Purdue e-Pubs, a service of the Purdue University Libraries. Please contact epubs@purdue.edu for additional information. 


\title{
Low Reynolds Number Flow through Nozzle-Diffuser Elements in Valveless Micropumps ${ }^{1}$
}

\author{
Vishal Singhal, Suresh V. Garimella ${ }^{2}$ and Jayathi Y. Murthy \\ Cooling Technologies Research Center \\ School of Mechanical Engineering, Purdue University \\ 585 Purdue Mall, West Lafayette, IN 47907-2088 USA
}

\begin{abstract}
Flow characteristics of low Reynolds number laminar flow through gradually expanding conical and planar diffusers were investigated. Such diffusers are used in valveless micropumps to effect flow rectification and thus lead to pumping action in one preferential direction. Four different types of diffuser flows are considered: fully developed and thin inlet boundary layer flows through conical and planar diffusers. The results from the numerical analysis have been quantified in terms of pressure loss coefficient. The variation of pressure loss coefficient with diffuser angle is presented for Reynolds numbers of 200, 500 and 1000. The pressure loss coefficients have been used to calculate the diffuser efficiency for two different types of nozzle-diffuser elements. The general trend of variation of pressure loss coefficient with diffuser angle was found to be similar to that for high Reynolds number turbulent flow. However, unlike at high Reynolds numbers, pressure loss coefficients at low Reynolds numbers vary significantly with Reynolds number. It was also observed that trends of variation in the pressure loss coefficient with Reynolds number are different for small and large diffuser angles. Also, at low Reynolds numbers, the pressure loss coefficients for a thin inlet boundary layer are not always smaller than those for fully developed inlet boundary layer, in contrast to the behavior for high Reynolds number flows. Contrary to past claims, flow rectification is shown to be indeed possible for laminar flows. The two different types of nozzle-diffuser elements considered led to pumping action in opposite directions. Further it was observed that flow rectification properties of both kinds of nozzle-diffuser elements improved with increasing Reynolds number.
\end{abstract}

Keywords: Valveless Micropump, Nozzle-Diffuser Flow, Low Reynolds Number Flow, Geometry Optimization, Computational Fluid Dynamics.

\footnotetext{
1 Submitted for publication in Sensors and Actuators A: Physical, June 2003, and in revised form, January 2004

2 To whom correspondence should be addressed: sureshg@ecn.purdue.edu, tel: (765) 494-5621; fax: (765) 494-0539
} 


\section{NOMENCLATURE}

$\begin{array}{ll}A & \text { area of cross-section } \\ C_{p} & \text { pressure recovery coefficient } \\ d & \text { characteristic dimension } \\ K & \text { pressure loss coefficient } \\ l & \text { length of nozzle or diffuser } \\ p & \text { static pressure } \\ Q & \text { volume flow rate } \\ r & \text { throat dimension } \\ \text { Re } & \text { Reynolds number } \\ \text { V } & \text { mean flow velocity } \\ \text { Greek Symbols }\end{array}$

$\varepsilon \quad$ flow rectification efficiency

$\theta \quad$ diffuser half-angle

$\eta \quad$ diffuser efficiency

$\rho \quad$ fluid density

$\Delta p \quad$ pressure loss

Subscripts and Superscripts

$+\quad$ positive direction

- $\quad$ negative direction

a cross-section a

$b \quad$ cross-section $\mathrm{b}$

d diffuser 


$\begin{array}{ll}\text { en } & \text { entrance } \\ \text { ex } & \text { exit } \\ n & \text { nozzle } \\ t & \text { total }\end{array}$

\section{INTRODUCTION}

A number of different micropump designs based on silicon microfabrication techniques have been presented over the last two decades [1-3]. A detailed review of these micropumping technologies was compiled very recently [4]. Amongst the various micropumping technologies, mechanical micropumps with vibrating diaphragms $[5,6]$ have generated the most interest. Although many novel pumping strategies such as pumps based on growing and collapsing bubbles [7], electrohydrodynamics [8], electroosmosis [9] and flexural plate waves [10] have also been presented, most of these pumps are not able to produce high flow rates (of the order of several hundred $\mu \mathrm{l} / \mathrm{min}$ to a few $\mathrm{ml} / \mathrm{min}$ ) which are easily achievable with mechanical micropumps. High flow rates can be the decisive factor in applications such as forced convective cooling of electronics devices.

Early efforts at fabricating vibrating diaphragm micropumps used diaphragm-type or cantilever-type active check valves. These valves suffer from many problems such as high pressure drops and wear and fatigue with long-term usage, which can cause leakage and severely limit the performance of the micropump. To overcome these problems, the use of fixed valves as substitutes for active check valves in vibrating-diaphragm micropumps was suggested [11-13]. For achieving flow rectification, such pumps utilize the differing flow (pressure drop) characteristics of fixed valves for flow in opposite directions. Valveless micropumps using two 
different types of fixed valves have been presented in the literature: i) nozzle-diffuser elements [11,12], and ii) valvular conduits [13]. Nozzle-diffuser elements, also known as dynamic passive valves [12], are the focus of this study.

A number of different valveless micropumps employing nozzle-diffuser elements have been discussed in the literature. These include piezoelectrically actuated [11], electromagnetically actuated [14], and bubble micropumps [15]. Use of nozzle-diffuser elements in magnetohydrodynamic micropumps has also been reported [16]. These pumps utilize the different pressure drop characteristics of flow through a nozzle and a diffuser to direct the flow in one preferential direction, and hence cause a net pumping action.

Additional benefits of nozzle-diffuser elements include the ease of manufacture using conventional silicon micromachining techniques, and the much higher flow rates achievable with vibrating diaphragm pumps employing such valves. The higher flow rates, in spite of the poorer flow rectification properties of such valves, stem from the possibility of using valveless micropumps at much higher frequencies as compared to micropumps with passive check valves. This is because passive check valves have a large response time, and pumps employing such valves cannot be excited to frequencies greater than a few hundred Hz. On the other hand, valveless micropumps can be excited to much higher frequencies $(\sim 10 \mathrm{kHz})$ and hence can achieve flow rates which are several orders of magnitude higher when compared to conventional passive check valve micropumps.

\section{Principle of Operation}

The operating principle of a valveless micropump is illustrated in Figure 1. The particular flow characteristics shown are for small nozzle-diffuser angles. In the expansion mode, as the volume of the pumping chamber increases, more fluid enters the pumping chamber from the element on 
the right which acts like a diffuser (and hence offers less flow resistance) than the element on the left, which acts like a nozzle. On the other hand, in the contraction mode, more fluid goes out of the element on the left which now acts as a diffuser, while the element on the right acts as a nozzle. Hence net fluid transport is achieved in the pumping chamber from right to left.

\section{NOZZLE-DIFFUSER ELEMENTS}

The volume flow rate of a valveless micropump depends on the rectification efficiency of the pump among other factors (such as amplitude and frequency of operation of the diaphragm). The rectification efficiency, $\varepsilon$, is the ratio of the volume of net fluid pumped to that crossing (entering or leaving) the pump in a given interval of time $\left(\varepsilon=\left(Q_{+}-Q_{-}\right) /\left(Q_{+}+Q_{-}\right)\right.$, see Figure 1$)$. The rectification efficiency of nozzle-diffuser micropumps reported in the literature is very low, generally between 0.01 and 0.2 . Since the rectification efficiency of these micropumps depends on the flow directing ability of the nozzle-diffuser elements, many studies have been directed at better understanding the fluid dynamic behavior and the flow rectification properties of nozzle-diffuser elements [11,14,17-20,23,24]. Different shapes of nozzle-diffuser elements have been considered in the literature. They can be broadly classified as spatial and planar. Spatial diffusers can be further divided into conical and pyramidal. These diffusers are schematically shown in Figure 2.

Gerlach and Wurmus [17] presented the first analysis of the performance of nozzle-diffuser elements. They microfabricated pyramidal nozzle-diffuser elements using anisotropic etching in $<100>$ silicon, which ensured that the half-angle of the diffuser would always be $35.26 \mathrm{deg}$. The dimension of the throat varied from 80 to $300 \mu \mathrm{m}$. From experiments on such stand-alone nozzle-diffuser elements, they concluded that the critical Reynolds number (Reynolds number in 
the present work and in the cited literature is based on the neck hydraulic diameter and mean velocity at the neck of the diffuser) was approximately 15 and that the flow was completely turbulent after a Reynolds number of 100. Since they found that the diffuser and nozzle had appreciably different flow characteristics only for Reynolds numbers greater than approximately 100, they further concluded that flow rectification occurs only for turbulent flow. Flow rectification efficiencies in the range of $0.05-0.15$ were reported.

Olsson et al. [18] conducted a pressure drop analysis of nozzle-diffuser elements. The total pressure drop across both the nozzle and the diffuser was divided into three parts: pressure drops due to sudden contraction at the inlet, gradual contraction or expansion along the length of the channel and sudden expansion at the exit, assuming negligible interference between these parts. Empirical values of pressure loss coefficients for these cases, obtained from macroscale experiments at Reynolds numbers in the range of 30,000-404,000 [21,22], were used to calculate the total pressure loss across the element for both nozzle and diffuser flow directions. Diffuser efficiency was defined as the ratio of pressure loss coefficients for the diffuser direction to that for the nozzle direction. Diffuser elements with half-oval shaped cross-sections were fabricated and tested. The experiments showed that the diffuser efficiency decreased as the angle of the diffuser decreased from 6.8 to $1.9 \mathrm{deg}$, but was not strongly affected by the length of the diffuser. The variation with angle was attributed to unsteady flow separation for diffusers with larger angles. Water and methanol were used as the working fluids and diffuser efficiency was found to be greater for methanol than for water. This was attributed to turbulent flow in methanol for Reynolds numbers in the range of $140-180$ as opposed to laminar flow in water for Reynolds numbers of 100-120.

Jiang et al. [14] analyzed the flow through a conical nozzle-diffuser element using different 
empirical correlations for pressure loss coefficients for low $(<50)$ and high $\left(>10^{5}\right)$ Reynolds number flows. They observed that for cone angles between 5 and $40 \mathrm{deg}$, the variation of diffuser efficiency with cone angle showed opposite trends in the two Reynolds number ranges, although the diffuser efficiency remained unchanged for a given cone angle in both Reynolds number ranges. They also concluded that the pumping direction would be different for the low and high Reynolds number flows. Their experiments on nozzle-diffuser elements of length $3 \mathrm{~mm}$, neck dimension $70 \mu \mathrm{m}$ and angles 5, 7.5 and $10 \mathrm{deg}$, showed that at Reynolds number close to 1800, diffuser efficiency decreased with increasing cone angle.

Gerlach [23] performed a pressure loss analysis similar to that in [18] for different diffuser angles and for sharp and well-rounded inlets to the nozzle-diffuser elements. Empirical values of pressure loss coefficients obtained at high Reynolds numbers [21] were again used, and flow rectification efficiencies calculated for different conical diffuser geometries. Experiments were performed on microfabricated pyramidal diffusers of half-angle 35.25 deg. Experimentally determined flow rectification values were compared to numerically determined values and the relatively large differences between the two were attributed to the different Reynolds numbers of the two flows.

In another study, Olsson et al. [24] used finite element simulations to analyze nozzle-diffuser elements, and compared the predictions to experiments. Their experimental results on various diffusers with cone angles 7, 9.8 and 13 deg showed that diffuser efficiency increased with decreasing cone angle and increasing diffuser length. It was observed that the laminar and turbulent flow simulations gave very similar results throughout the Reynolds number range considered (approximately up to 1400). The transition Reynolds number was calculated to be 400. 
From the review of the literature above, it is clear that fluid flow through nozzle-diffuser elements for micropumps is not well understood. A number of conflicting results regarding the nature of the flow (laminar or turbulent) and variation of diffuser efficiency with cone angle and diffuser length have been reported. For instance, Olsson et al. [18], Gerlach and Wurmus [17] and Gerlach [23] found that turbulent flow through the diffuser led to a better flow-directing ability than laminar flow, while in [24] Olsson et al. reported from their numerical results that laminar and turbulent flow led to very similar flow characteristics. Further, Olsson et al. [18] experimentally showed that the diffuser efficiency decreases with decreasing angle but is independent of diffuser length. In [24], however, they reported that diffuser efficiency increased with decreasing cone angle and increasing diffuser length. Further, Jiang et al. [14] found from experiments that diffuser efficiency decreases with decreasing angle and Gerlach [23] showed that the diffuser efficiency increases with the length of the diffuser.

Moreover, all the pressure drop analyses undertaken for calculating the diffuser efficiency have used empirical pressure loss coefficients obtained at Reynolds numbers in the range of 30,000 and higher [18,23], while the Reynolds number for flow through micropumps rarely exceeds 5000, and is generally in the range of 100-500. Indeed, Jiang et al. [14] showed that the diffuser efficiency showed different trends with cone angle at low $(<50)$ and high $\left(>10^{5}\right)$ Reynolds numbers.

Clearly, there is a need to better understand the flow behavior through nozzle-diffuser elements at low Reynolds numbers. The present study addresses this need. The variation of pressure losses with the diffuser angle through gradually expanding diffusers is determined numerically using a finite volume approach. Conical and planar diffuser cross-section shapes are considered. In addition, smooth (rounded) and sharp entrances, which respectively cause the inlet boundary layer to be fully developed and thin, are considered to assess their impact on pressure losses. The 
analysis considers flow Reynolds numbers of 200, 500 and 1000. The results from the numerical analyses are used to characterize the flow-directing ability of different diffuser elements. It may be noted that flow characteristics for low Reynolds number flow in a nozzle (as opposed to a diffuser) are not expected to be significantly different from those at the higher Reynolds numbers [24]; hence, nozzle flow is not considered in this work, and the focus is restricted to diffuser flow.

\section{THEORETICAL ANALYSIS}

\section{Pressure Loss Coefficient}

The pressure loss coefficient for flows through a gradually contracting nozzle, a gradually expanding diffuser, or a sudden expansion or contraction in an internal flow system is defined as the ratio of pressure drop across the device to the velocity head upstream of the device:

$$
K=\frac{\Delta p}{\rho \mathrm{v}^{2} / 2}
$$

For flow through a gradually expanding diffuser (Figure 3) or a gradually contracting nozzle, the pressure loss coefficient can be calculated as follows. For flow in the diffuser direction (from cross-section a to b in Figure 3), the incompressible steady-flow energy equation reduces to

$$
p_{a}+\frac{\rho}{2} \mathrm{v}_{a}^{2}=p_{b}+\frac{\rho}{2} \mathrm{v}_{b}^{2}+\Delta p_{d}
$$

Hence, the pressure loss coefficient can be written as

$$
K_{d}=\frac{\Delta p_{d}}{\rho \mathrm{v}_{a}^{2} / 2}=\frac{\left(p_{a}-p_{b}\right)}{\rho \mathrm{v}_{a}^{2} / 2}+\left(1-\frac{\mathrm{v}_{b}^{2}}{\mathrm{v}_{a}^{2}}\right)
$$

Introducing the pressure recovery coefficient $C_{p}=\left(p_{b}-p_{a}\right) /\left(\rho \mathrm{v}_{a}^{2} / 2\right)$ and using the continuity equation $A_{a} \mathrm{v}_{a}=A_{b} \mathrm{v}_{b}, K_{d}$ for spatial diffusers (e.g., conical and pyramidal) can be written as 


$$
K_{d}=1-\frac{d_{a}^{4}}{d_{b}^{4}}-C_{p}
$$

since $A \propto d^{2}$. While for planar diffusers, $A \propto d$ and hence $K_{d}$ is given by

$$
K_{d}=1-\frac{d_{a}^{2}}{d_{b}^{2}}-C_{p}
$$

Hence for a given diffuser geometry, the pressure loss coefficient can be calculated from the pressure drop and the mean velocity at the neck. Similarly, for flow in the nozzle direction (from cross-section b to a in Figure 3), the pressure loss coefficient is given by,

$$
K_{n}=\frac{\Delta p_{n}}{\rho v_{b}^{2} / 2}
$$

Pressure loss coefficients for flow through sudden expansions and contractions can similarly be calculated.

\section{Diffuser Efficiency}

The diffuser efficiency of a nozzle-diffuser element is defined as the ratio of the total pressure loss coefficient for flow in the nozzle direction to that for the flow in the diffuser direction:

$$
\eta=\frac{K_{n, t}}{K_{d, t}}
$$

Hence, $\eta>1$ will cause a pumping action in the diffuser direction (Figure 1) in a valveless micropump, while $\eta<1$ will lead to pumping action in the nozzle direction. The case where $\eta=$ 1 corresponds to equal pressure drops in both the nozzle and the diffuser directions, leading to no flow rectification. In Equation (6), the total pressure loss coefficients for both the diffuser and nozzle directions can be divided into three parts: (i) losses due to sudden contraction at the entrance, (ii) losses due to gradual contraction or expansion through the length of the nozzle/diffuser, and (iii) losses due to sudden expansion at the exit. The total pressure drop in the 
diffuser direction can thus be written as

$$
\Delta p_{d, t}=\Delta p_{d, e n}+\Delta p_{d}+\Delta p_{d, e x}
$$

Therefore, the total pressure loss coefficient for the diffuser can be calculated as

$$
\begin{aligned}
K_{d, t} & =\frac{\Delta p_{d, t}}{\rho v_{a}^{2} / 2}=\frac{\Delta p_{d, e n}}{\rho v_{a}^{2} / 2}+\frac{\Delta p_{d}}{\rho v_{a}^{2} / 2}+\frac{\Delta p_{d, e x}}{\rho v_{b}^{2} / 2} \frac{\rho v_{b}^{2} / 2}{\rho v_{a}^{2} / 2} \\
& =K_{d, e n}+K_{d}+K_{d, e x} \frac{A_{a}^{2}}{A_{b}^{2}}
\end{aligned}
$$

Similarly, the total pressure loss coefficient for the nozzle (with respect to pressure head at the neck) is

$$
K_{n, t}=\left(K_{n, e n}+K_{n}\right) \frac{A_{a}^{2}}{A_{b}^{2}}+K_{n, e x}
$$

Therefore, diffuser efficiency can be written as,

$$
\eta=\frac{K_{n, t}}{K_{d, t}}=\frac{\left(K_{n, e n}+K_{n}\right) A_{a}^{2} / A_{b}^{2}+K_{n, e x}}{K_{d, e n}+K_{d}+K_{d, e x} A_{a}^{2} / A_{b}^{2}}
$$

\section{Flow Rectification Efficiency}

The flow rectification efficiency of a valveless micropump is the measure of the ability of the pump to direct the flow in one preferential direction. It can be expressed as

$$
\varepsilon=\frac{Q_{+}-Q_{-}}{Q_{+}+Q_{-}}
$$

in which $Q$ is flow rate and subscripts + and - refer to flow in the forward and the backward directions, respectively. A higher $\varepsilon$ corresponds to better flow rectification. In particular, when there is no flow rectification, equal amounts of fluid move in both directions and $\varepsilon=0$, while for perfect rectification, flow is only in one direction and $\varepsilon=1$. The flow rectification efficiency of a valveless micropump is related to the diffuser efficiency of the nozzle-diffuser elements. As the diffuser efficiency departs from a value of 1, i.e., as the difference between $K_{n, t}$ and $K_{d, t}$ 
increases, $\varepsilon$ for the micropump also increases.

\section{NUMERICAL SIMULATIONS}

The problem of determining the pressure drop and the average neck velocity in a diffuser is solved numerically using the finite volume method. The commercially available software package FLUENT [25] is used to model and solve the problem. Conical and planar diffusers are considered; the effects of both sharp and smooth entrance conditions are studied. Smooth edges will cause the flow entering the diffuser to be relatively fully developed, while sharp edges will lead to thin boundary layers at the inlet cross-section. Steady-state laminar flow simulations are carried out for Reynolds numbers of 200, 500 and 1000. Care was taken to adjust the inlet velocity such that the Reynolds number was within $1 \%$ of these values. Simulations are performed for diffuser half-angles varying from $2.5 \mathrm{deg}$ to $70 \mathrm{deg}$ in increments of $2.5 \mathrm{deg}$. The particular geometries modeled for the four cases considered - fully developed and thin boundary layer inlet flow for the conical and planar diffusers - are shown in Figure 4.

Mesh-independence was verified for all the cases considered, by refining the mesh until the change in results was within $1 \%$. Finer meshes were also needed as the half-angle of the nozzle-diffuser elements was increased. For the conical element, the $(\mathrm{z} \times \mathrm{r})$ mesh was increased from $300 \times 40$ (fully developed inlet) and $200 \times 40$ (thin inlet) at the smallest half angles to $300 \times$ 120 (fully developed inlet) and $200 \times 120$ (thin inlet) for the largest; for the planar geometry the transverse mesh $(\mathrm{x} \times \mathrm{y})$ was increased from $40 \times 40$ to $120 \times 120$ as the half-angle increased.

The conical diffusers are modeled as being axi-symmetric about the horizontal axis. For the planar diffuser, symmetry boundary conditions are used to model only a quarter of the cross-section. The flow is considered incompressible. Uniform velocity inlet and uniform 
pressure outlet boundary conditions are applied at cross-sections 1 and 4, respectively, for all the four cases. In addition, no-slip boundary conditions are imposed at the walls.

Steady-state analysis was used to calculate the pressure loss coefficients even though the flow in the nozzle-diffuser elements would be oscillating (potentially at high frequencies). The pressure loss coefficients for this transient flow may be different from the steady-state values presented here. However, transient loss coefficients would depend on a number of variables specific to the design, such as the frequency of oscillation, and the rate and profile of variation of the mean flow Reynolds number, in addition to the parameters considered here (absolute value of the Reynolds number, and angle and shape of the nozzle-diffuser elements). Steady-state pressure loss coefficients are, therefore, believed to be useful for preliminary design of the nozzle-diffuser elements.

\section{Model Validation}

The numerical model was validated by simulating turbulent flow at high Reynolds numbers in conical diffusers for fully developed and thin inlet boundary layer flows. The two equation $\kappa-\varepsilon$ model in FLUENT was used to model the turbulent flow for a Reynolds number of 100,000. The numerical values of the predicted pressure loss coefficients are compared to the experimental values available for turbulent flows in the literature. The comparison is shown in Table 1. The experimental data for a thin inlet boundary layer are from [21] and those for a fully developed boundary layer are from [26]. The experimental values are linearly interpolated between data read from charts.

While there is reasonable agreement between the predicted and experimental results, the predictions are lower in general, especially at the larger angles. This may be attributed to inadequate handling of separation by the $\kappa-\varepsilon$ model and additional pressure losses in the 
experimental set-up due to wall friction and roughness effects which are not accounted for in the predictions.

\section{RESULTS AND DISCUSSION}

Pressure loss coefficients $\left(K_{d}\right)$ for fully developed and thin inlet boundary layer flows in a conical diffuser are plotted in Figures 5(a) and (b), respectively, as a function of cone half-angle for Reynolds numbers (Re) of 200, 500 and 1000 and for high Reynolds number turbulent flow. The pressure loss coefficients at low Reynolds number were calculated using Equation (3) in combination with the pressure drop and the average neck velocity obtained from the numerical simulations. The turbulent flow curves plotted were obtained from experimental results compiled in Ref. [21]. For both inlet flow conditions, the variation of $K_{d}$ with cone angle follows the general trend observed for high Reynolds number flows ( $\operatorname{Re}>30,000)$, although $K_{d}$ values obtained here are greater and smaller than those for high Reynolds number flows at small and large cone half-angles, respectively. Moreover, at the low Reynolds numbers considered, $K_{d}$ for a given diffuser angle varies significantly with Reynolds number, especially at small cone angles. In contrast, $K_{d}$ for high Reynolds number flows does not vary with Reynolds number.

For fully developed inlet boundary layer flow, $K_{d}$ is the lowest for $\operatorname{Re}=1000$ and the highest

for $\operatorname{Re}=200$, when the cone half-angle is less than approximately $5 \mathrm{deg}$. For larger half-angles, the opposite is true, i.e. $K_{d}$ is lowest for $\mathrm{Re}=200$ and highest for $\mathrm{Re}=1000$. For small cone angles, the loss coefficients decrease with increasing Re, while at large cone angles, they increase with increasing Re. The high loss coefficients for small diffuser angles at low Re are believed to be due to the dominance of viscous forces in these very ordered flows. As the cone angle increases, flow separation occurs, which is associated with higher loss coefficients (higher than the 
viscous contributions). Since flow separation is more dominant for higher Re, loss coefficients at the larger cone angles are also greater for larger Re. The same phenomena are also observed for the thin inlet boundary layer flow, although in this case, viscous losses seem to dominate up to cone half-angles of 15 to $20 \mathrm{deg}$.

For the case of the fully developed inlet boundary layer, back flow at the outlet boundary (cross-section 4 in Figure 4a), was first observed for the cone half-angle of $7.5 \mathrm{deg}$ at $\operatorname{Re}=500$ and 1000, and for the half-angle of $10 \mathrm{deg}$ at $\mathrm{Re}=200$. Similarly, for the thin inlet boundary layer, back flow (at cross-section 4 in Figure 4b) was first observed for the cone half-angle of 12.5 deg at $\operatorname{Re}=500$ and 1000 and for the half-angle of 15 deg at $\operatorname{Re}=200$. The conditions under which back flow starts correspond to the cone angle beyond which the higher Reynolds number flow has the greater loss coefficients. This further strengthens the proposed reasoning for the opposing trends of variation in the pressure loss coefficient with Reynolds number for small and large cone angles.

Comparing the numerical values of $K_{d}$ for the fully developed and thin inlet boundary layer flows, it can be observed that for small cone angles, $K_{d}$ is smaller for the fully developed boundary layer and vice versa ( $K_{d}$ is smaller for the thin inlet boundary layer flows for large cone angles). Also, this behavior is peculiar to low Reynolds number flow. At high Reynolds numbers, $K_{d}$ for the fully developed inlet boundary layer flow is smaller than that for thin inlet boundary layers for all cone angles. The higher $K_{d}$ for thin inlet boundary layer flows might be attributable to an additional pressure drop due to the boundary layer development in these flows, which is absent for fully developed inlet boundary layers.

Pressure loss coefficients for the fully developed and thin inlet boundary layer flow through a planar diffuser are plotted in Figures 6(a) and (b), respectively, as a function of the diffuser angle. 
Observations made with reference to Figure 5 for flow through conical diffusers apply to Figure 6 as well. It is interesting to note that not only the trends of variation but also the numerical values for fully developed flow through conical and planar diffusers are very close, especially for the fully developed inlet boundary layer flow. It has been reported in the literature that at high Reynolds numbers, the maximum pressure recovery coefficients for conical and flat diffusers are approximately the same. However, the pressure recovery coefficients for the same length of diffuser were not always identical [22].

In the following paragraphs, two different types of nozzle-diffuser elements, which have been used in valveless micropumps presented in the literature, are analyzed. The pressure loss coefficients obtained using the numerical simulations presented above are used to calculate the flow rectification efficiency possible with these nozzle-diffuser elements.

The two representative nozzle-diffuser element types considered are as follows. A Type 1 nozzle-diffuser element is conical with a rounded inlet, a sharp outlet and a cone half-angle of 2.5 deg. A Type 2 nozzle-diffuser element is conical with a sharp inlet, a sharp outlet and a cone half-angle of $35 \mathrm{deg}$. These two combinations of nozzle-diffuser elements have been considered in past studies, Type 1 in $[11,18]$ and Type 2 in $[12,23]$.

In order to calculate diffuser efficiencies for these two types of nozzle-diffuser elements, the pressure loss coefficients for operation as a nozzle under sudden contraction/expansion and gradual contraction are required. Values for these loss coefficients are available in the literature [24] and are reproduced in Table 2 for both types of nozzle-diffuser elements. Although these coefficients for nozzle flow were obtained from experiments at large Reynolds numbers, they are not expected to differ much at low Reynolds numbers [24], and can be used in the present computations. 
The pressure loss coefficients for flow in a gradually expanding diffuser, for both types of nozzle-diffuser elements, along with the total loss coefficients in the two directions and the diffuser efficiencies, are given in Table 3 for $\operatorname{Re}=200,500$ and 1000. The diffuser efficiencies for both the nozzle-diffusers elements are calculated for $l / r=5$ (Figure 4), using Equations (8) (10) and the numerical values in Table 2.

Since the diffuser efficiency $\eta$, for the Type 1 nozzle-diffuser elements is greater than 1 , the pumping action would be in the diffuser direction (from cross-section a to b in Figure 3). Also, the volume flow rate of the pumped fluid would increase with increasing Reynolds number. On the other hand, for Type 2 nozzle-diffuser elements, fluid would be pumped in the opposite (nozzle) direction. Here as well, the volume flow rate would increase with increasing Reynolds number, since the flow rectification efficiency increases as $\eta$ departs from a value of 1 as discussed earlier in this paper. Hence, while a pumping action is effected for both types of nozzle-diffuser elements, the flow rectification efficiency and the volume flow rate would be higher for Type 1 nozzle-diffuser elements, as was reported earlier [23, 24].

\section{CONCLUSIONS}

The following key conclusions may be drawn from the results of the present work:

1. It is found that the general trends of variation of pressure loss coefficient with diffuser angle for both fully developed and thin inlet boundary layer flows through gradually expanding diffusers are similar to that for high Reynolds number turbulent flow. However, unlike high Reynolds number flows, pressure loss coefficients for low Reynolds number laminar flows are a strong function of the flow Reynolds number, especially at small diffuser angles. 
2. The variation of pressure loss coefficient with Reynolds number follows opposite trends for small and large diffuser angles. Hence, the Reynolds number of the flow should be considered in the design of micropumps employing such valves.

3. It is observed that unlike high Reynolds number flows, the pressure loss coefficients for thin inlet boundary layer flows are not always smaller than those for fully developed inlet boundary layer flows.

4. Flow rectification in nozzle-diffuser elements is achievable in laminar flow. This is in contrast to earlier reports in the literature $[17,18,23]$.

5. For the two types of conical nozzle-diffuser elements considered, one with a rounded inlet, sharp outlet and diffuser half-angle of 2.5 deg and other with a sharp inlet, sharp outlet and diffuser half-angle of $35 \mathrm{deg}$, flow rectification improved with increasing Reynolds number.

6. Both the numerical values as well as the trends of variation for fully developed flow through conical and planar diffusers are very similar.

In ongoing work, planar nozzle-diffuser elements are being studied further, since they are easier to fabricate using silicon microfabrication techniques. The effect of variation of length on the pressure loss coefficients and flow rectification is being studied. The complete micropump will also be analyzed using deforming grids, to relax the assumption regarding negligible interference between different parts of the nozzle-diffuser elements and the pumping chamber. 


\section{REFERENCES}

[1] P. Gravesen, J. Branebjerg, O.S. Jensen, Microfluidics - a review, Journal of Micromechanics \& Microengineering, 3 (1993), 168-182.

[2] S. Shoji, M. Esashi, Microflow devices and systems, Journal of Micromechanics and Microengineering, 4 (1994), 157-171.

[3] G.T.A. Kovacs, Micromachined Transducers Sourcebook, New York, McGraw Hill, 1998, pp. 839-855.

[4] V. Singhal, S.V. Garimella, A. Raman, Microscale Pumping Technologies for Microchannel Cooling Systems, Applied Mechanics Reviews (in press)

[5] H.T.G. van Lintel, F.C.M. van De Pol, S. Bouwstra, S., A piezoelectric micropump based on micromachining of silicon, Sensors and Actuators, 15 (1988), 153-167.

[6] R. Zengerle, J. Ulrich, S. Kluge, M. Richter, A. Richter, A bidirectional silicon micropump, Sensors and Actuators A: Physical, 50 (1995), 81-86.

[7] T.K. Jun, C.-J. Kim, Valveless pumping using traversing vapor bubbles in microchannels, Journal of Applied Physics, 83 (1998), 5658-5664.

[8] S.F. Bart, L.S. Tavrow, M. Mehregany, J.H. Lang, Microfabricated electrohydrodynamic pumps, Sensors and Actuators A: Physical, A21-A23 (1990), 193-197.

[9] A. Manz, C.S. Effenhauser, N. Burggraf, D.J. Harrison, K. Seiler, K. Fluri, Electroosmotic pumping and electrophoretic separations for miniaturized chemical analysis systems, Journal of Micromechanics and Microengineering, 4 (1994), pp. 257-265.

[10] R.M. Moroney, R.M. White, R.T. Howe, Microtransport induced by ultrasonic Lamb waves, Applied Physics Letters, 59 (1991), 774-776.

[11] E. Stemme, G. Stemme, A valveless diffuser/nozzle-based fluid pump, Sensors and Actuators A: Physical, 39 (1993), 159-167.

[12] T. Gerlach, M. Schuenemann, H. Wurmus, H., A new micropump principle of the reciprocating type using pyramidic micro flowchannels as passive valves, Journal of Micromechanics and Microengineering, 5 (1995), 199-201.

[13] F.K. Forster, R.L. Bardell, M.A. Afromowitz, N.R. Sharma, A. Blanchard, Design, fabrication and testing of fixed-valve micro-pumps, Proceedings of the ASME Fluids Engineering Division, FED 234 (1995), 39-44.

[14] X.N. Jiang, Z.Y. Zhou, X.Y. Huang, Y. Li, Y. Yang, C.Y. Liu, Micronozzle/diffuser flow and its application in micro valveless pumps, Sensors and Actuators A: Physical, 70 (1998), 81-87.

[15] J.-H. Tsai, L. Lin, A thermal bubble actuated micro nozzle-diffuser pump, Proceedings of the IEEE Micro Electro Mechanical Systems (MEMS), Interlaken, Switzerland, (2001), 409-412.

[16] K.-H. Heng, L. Huang, W. Wang, M.C. Murphy, Development of a diffuser/nozzle type micropump based on magnetohydrodynamic (MHD) principle, Proceedings of SPIE - the International Society for Optical Engineering, 3877 (1999), 66-73.

[17] T. Gerlach, H. Wurmus, Working principle and performance of the dynamic micropump, Sensors and Actuators A: Physical, 50 (1995), 135-140. 
[18] A. Olsson, G. Stemme, E. Stemme, Diffuser-element design investigation for valve-less pumps, Sensors and Actuators A: Physical, 57 (1996), 137-143.

[19] M. Heschel, M. Mullenborn, S. Bouwstra, Fabrication and characterization of truly 3-D diffuser/nozzle microstructures in silicon, Journal of Microelectromechanical Systems, 6 (1997), 41-47.

[20] M. Koch, A.G.R. Evans, A. Brunnschweiler, The dynamic micropump driven with a screen printed PZT actuator, Journal of Micromechanics and Microengineering, 8 (1998), 119-122.

[21] F.M. White, Fluid Mechanics, New York, McGraw-Hill, 1994, 335-341.

[22] P.W. Runstadler, Jr., F.X. Dolan, R.C. Dean, Diffuser Data Book, Hanover, NH, Creare Inc., 1975.

[23] T. Gerlach, Microdiffusers as dynamic passive valves for micropump applications, Sensors and Actuators A: Physical, 69 (1998), 181-191.

[24] A. Olsson, G. Stemme, E. Stemme, Numerical and experimental studies of flat-walled diffuser elements for valve-less micropumps, Sensors and Actuators A: Physical, 84 (2000), 165-175.

[25] Fluent, Fluent 6 User's Guide, Fluent Inc, Lebanon, NH, 2000.

[26] D.S. Miller, Internal Flow Systems, BHRA Fluid Engineering, 1978. 


\section{List of Tables}

Table 1. Comparison of numerical and experimental pressure loss coefficients [21, 26] for large Reynolds number turbulent flow.

Table 2. Pressure loss coefficients for entrance, exit and nozzle flow for the two types of nozzle-diffuser elements.

Table 3. Total pressure loss coefficients and diffuser efficiency for nozzle-diffuser elements of Types 1 and 2 at different Reynolds numbers. 
Table 1. Comparison of numerical and experimental pressure loss coefficients $[21,26]$ for large Reynolds number turbulent flow.

\begin{tabular}{|c|c|c|c|}
\hline \multirow{2}{*}{$\begin{array}{c}\text { Angle } \\
\text { (deg) }\end{array}$} & \multirow{2}{*}{$\begin{array}{c}\text { Inlet Boundary } \\
\text { Layer }\end{array}$} & \multicolumn{2}{|c|}{$K_{d}$} \\
\cline { 3 - 4 } & Numerical & Experimental \\
\hline 5 & Fully Developed & 0.061 & 0.06 \\
\hline 70 & Fully Developed & 0.940 & 1.20 \\
\hline 5 & Thin & 0.115 & 0.13 \\
\hline 70 & Thin & 0.824 & 1.02 \\
\hline
\end{tabular}


Table 2. Pressure loss coefficients for entrance, exit and nozzle flow for the two types of nozzle-diffuser elements.

\begin{tabular}{|c|c|c|c|c|c|}
\hline & $K_{d, e n}$ & $K_{d, e x}$ & $K_{n, e n}$ & $K_{n}$ & $K_{n, e x}$ \\
\hline Type 1 & 0.05 & 1.00 & 0.40 & 0.03 & 1.00 \\
\hline Type 2 & 0.40 & 1.00 & 0.40 & 0.07 & 1.00 \\
\hline
\end{tabular}


Table 3. Total pressure loss coefficients and diffuser efficiency for nozzle-diffuser elements of Types 1 and 2 at different Reynolds numbers.

\begin{tabular}{|c|c|c|c|c|}
\hline \multicolumn{2}{|c|}{} & $\mathrm{Re}=200$ & $\mathrm{Re}=500$ & $\mathrm{Re}=1000$ \\
\hline \multirow{4}{*}{ Type 1 } & $K_{d}$ & 0.59 & 0.39 & 0.33 \\
\cline { 2 - 5 } & $K_{n, t}$ & 1.19 & 1.19 & 1.19 \\
\cline { 2 - 5 } & $K_{d, t}$ & 1.09 & 0.89 & 0.83 \\
\cline { 2 - 5 } & $\eta$ & 1.09 & 1.34 & 1.43 \\
\hline \multirow{4}{*}{ Type 2 } & $K_{d}$ & 0.77 & 0.81 & 0.85 \\
\cline { 2 - 5 } & $K_{n, t}$ & 1.00 & 1.00 & 1.00 \\
\cline { 2 - 5 } & $K_{d, t}$ & 1.17 & 1.21 & 1.25 \\
\cline { 2 - 5 } & $\eta$ & 0.85 & 0.83 & 0.80 \\
\hline
\end{tabular}




\section{List of Figures}

Figure 1. Flow rectification in a valveless micropump: (a) Expansion mode (increasing volume of the pumping chamber) and, (b) contraction mode (decreasing volume of the pumping chamber). The thicker arrows imply higher volume flow rates.

Figure 2. Schematic of (a) conical, (b) pyramidal and (c) planar nozzle-diffuser elements.

Figure 3. Schematic of a nozzle-diffuser element.

Figure 4. Geometries modeled to simulate (a) fully developed, and (b) thin inlet boundary layer inlet flow in a conical diffuser (using an axi-symmetric model), and (c) fully developed, and (d) thin inlet boundary layer inlet flow in a planar diffuser.

Figure 5. Plot of variation of pressure loss coefficient in a conical diffuser with half-angle of the cone for $\operatorname{Re}=200,500,1000$ and $>30,000$ for (a) fully developed inlet boundary layer; and (b) thin inlet boundary layer.

Figure 6. Plot of variation of pressure loss coefficient in a planar diffuser with half-angle of the cone for $R e=200,500$ and 1000 for (a) fully developed inlet boundary layer; and (b) thin inlet boundary layer. 


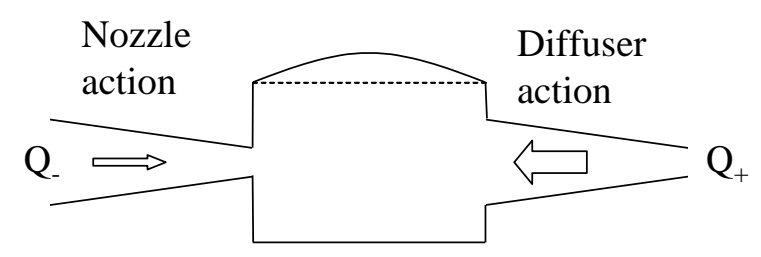

(a)

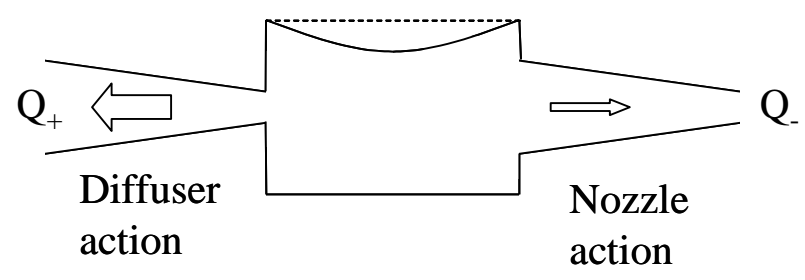

(b)

Figure 1. Flow rectification in a valveless micropump: (a) Expansion mode (increasing volume of the pumping chamber) and, (b) contraction mode (decreasing volume of the pumping chamber). The thicker arrows imply higher volume flow rates. 


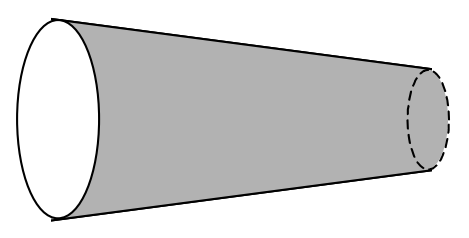

(a)

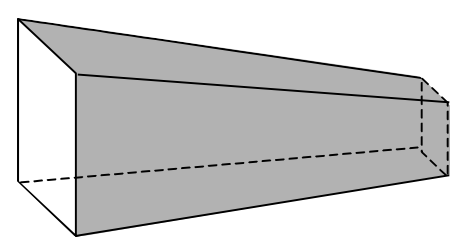

(b)

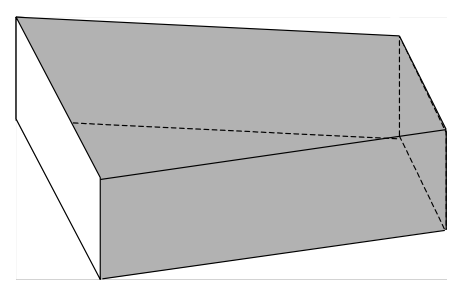

(c)

Figure 2. Schematic of (a) conical, (b) pyramidal and (c) planar nozzle-diffuser elements. 


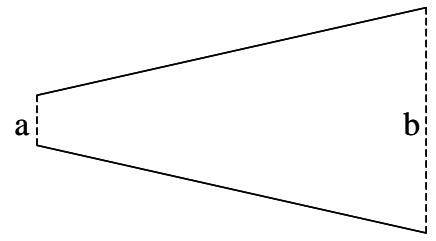

Figure 3. Schematic of a nozzle-diffuser element. 


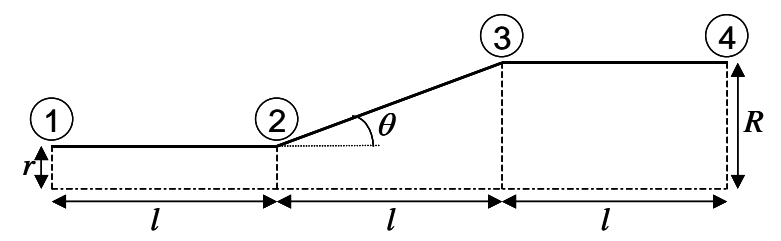

(a)

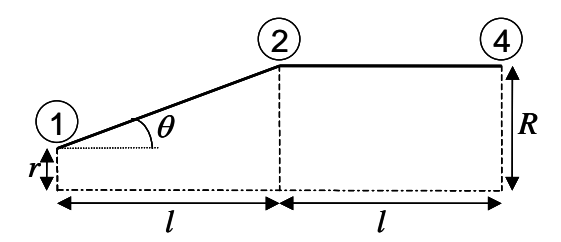

(b)

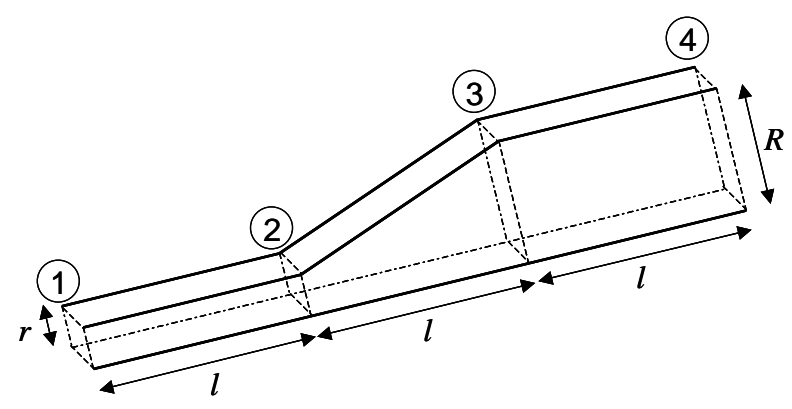

(c)

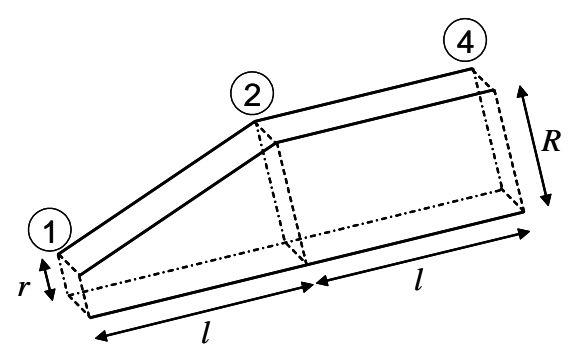

(d)

Figure 4. Geometries modeled to simulate (a) fully developed, and (b) thin inlet boundary layer inlet flow in a conical diffuser (using an axi-symmetric model), and (c) fully developed, and (d) thin inlet boundary layer inlet flow in a planar diffuser. 


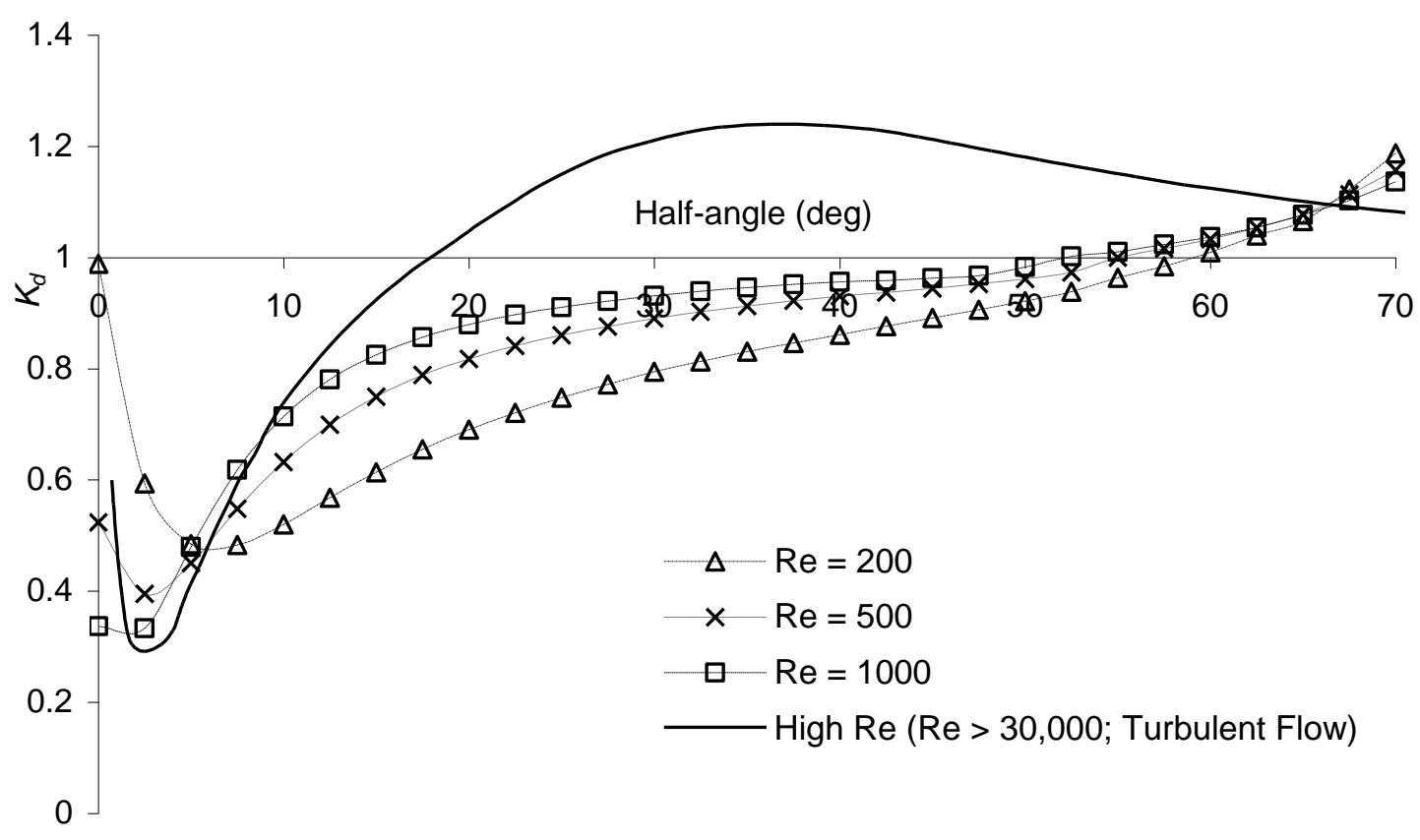

(a)

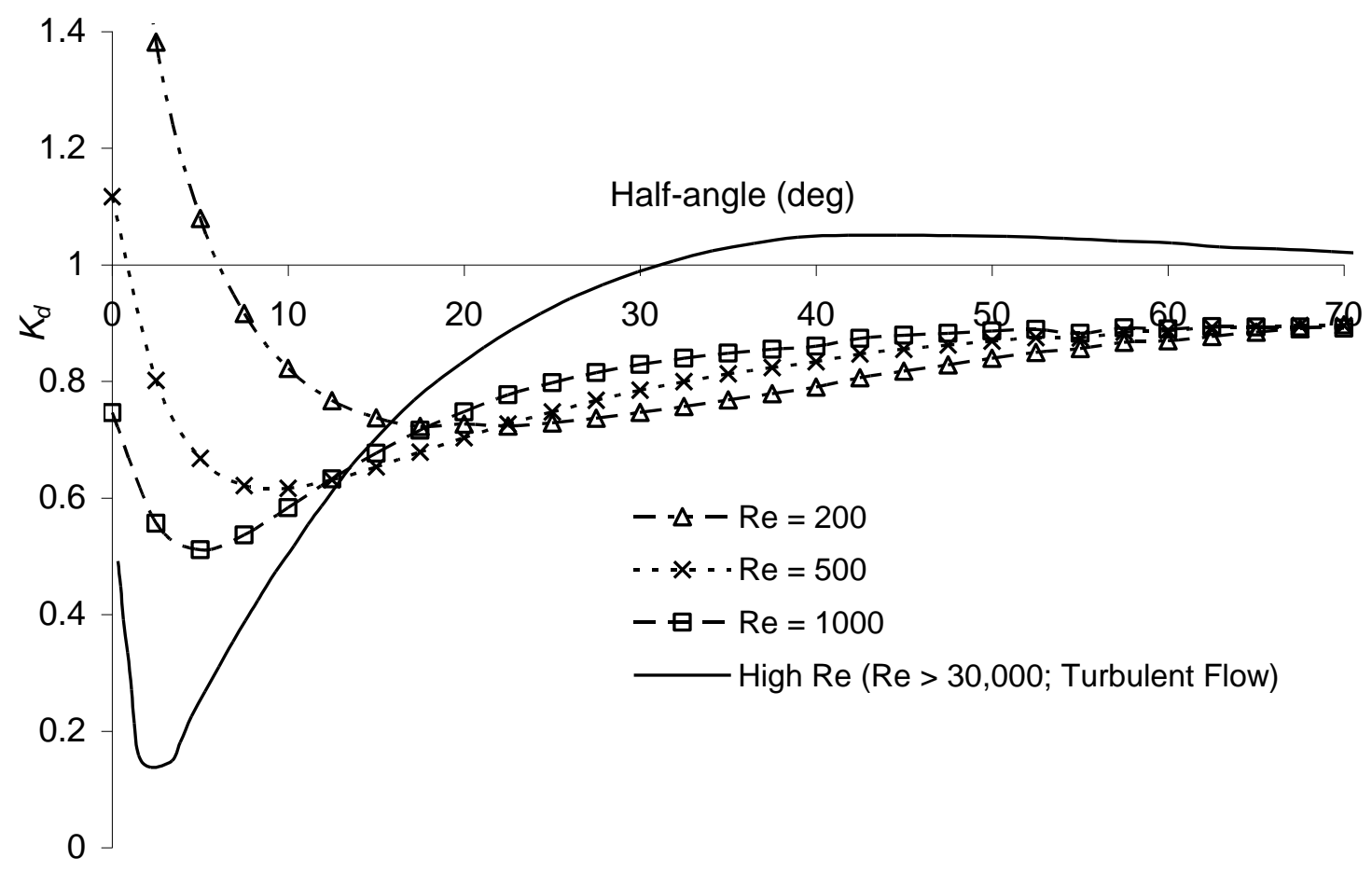

(b)

Figure 5. Plot of variation of pressure loss coefficient in a conical diffuser with half-angle of the cone for $\operatorname{Re}=200,500,1000$ and $>30,000$ for (a) fully developed inlet boundary layer; and (b) thin inlet boundary layer. 


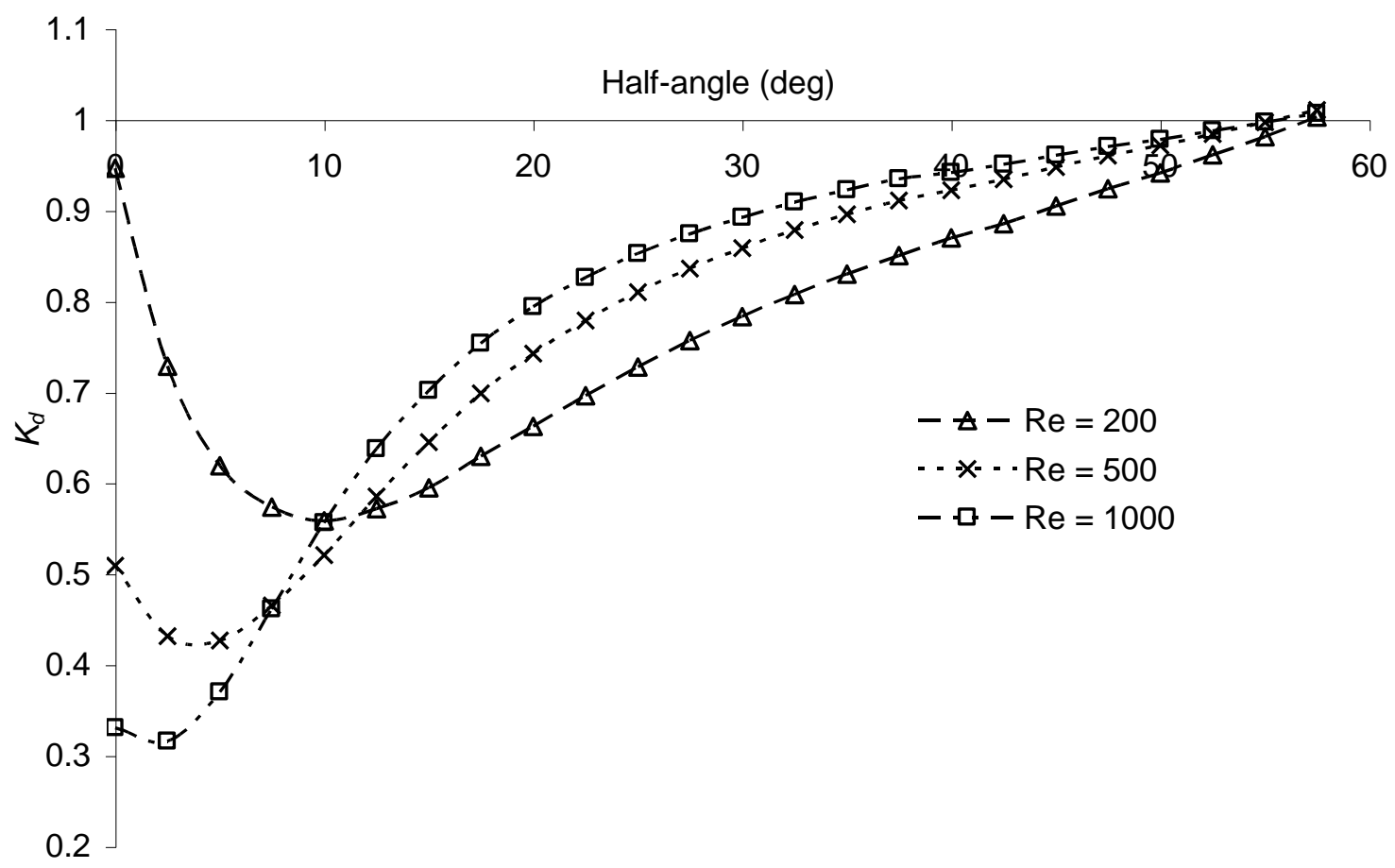

(a)

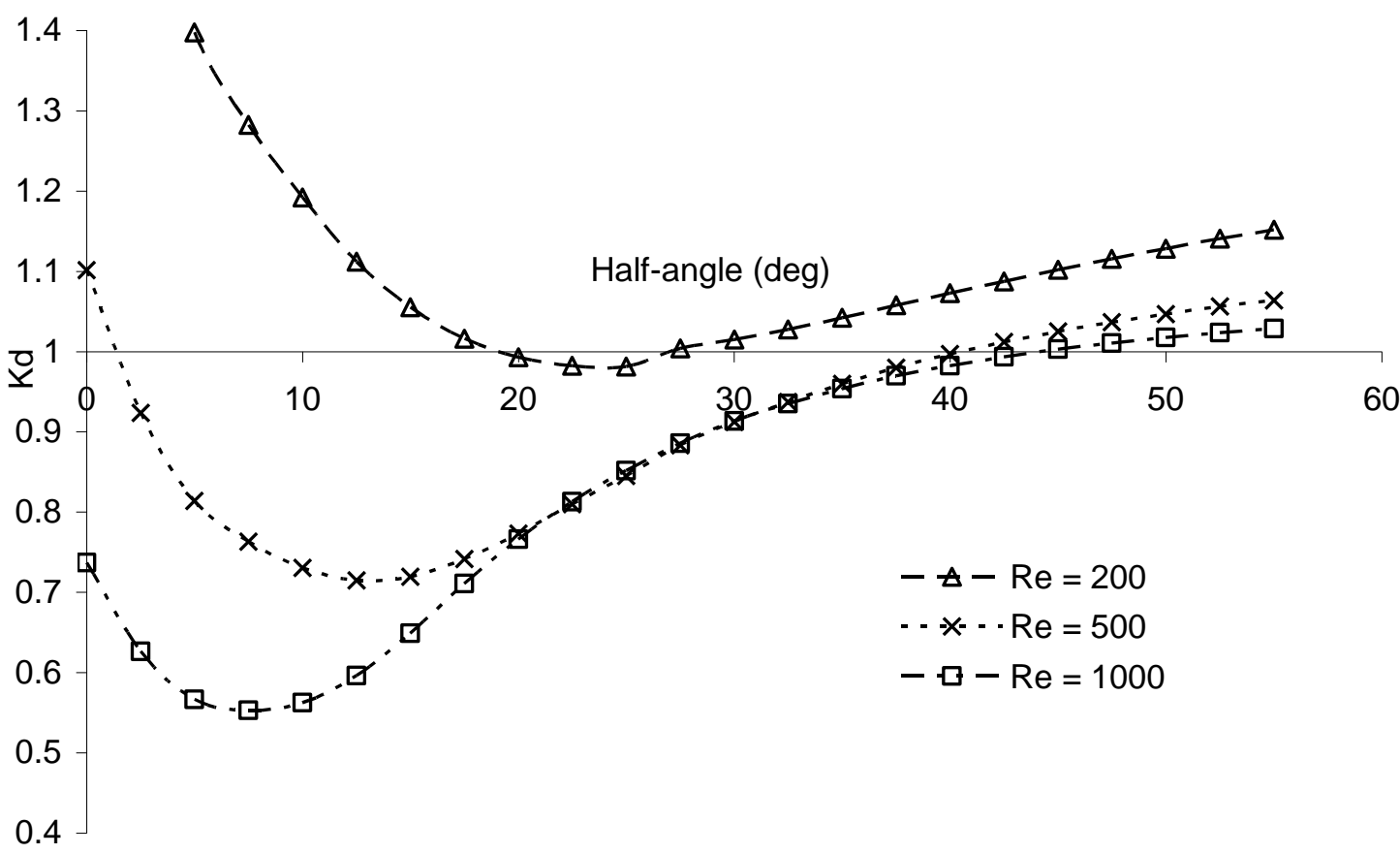

(b)

Figure 6. Plot of variation of pressure loss coefficient in a planar diffuser with half-angle of the cone for $\operatorname{Re}=200,500$ and 1000 for (a) fully developed inlet boundary layer; and (b) thin inlet boundary layer. 